\title{
Type 1 diabetes mellitus masking primary antibody deficiency
}

\author{
S L Johnston, P F Virgo, D J Unsworth
}

\begin{abstract}
A patient with a history of recurrent cutaneous and pulmonary infections, nephrotic syndrome, and an established diagnosis of type 1 diabetes was found to have unsuspected and unrecognised primary immunodeficiency. On review of the case, previous investigations pointed to the correct diagnosis over 10 years earlier. This combination of diagnoses has not previously been reported. The patient is now well on replacement intravenous immunoglobulin therapy, urinary loss of IgG having been specifically excluded before treatment. This case highlights how antibody deficiency can easily be missed despite an obvious infection history unless results are interpreted carefully and in context.

(f Clin Pathol 2000;53:236-237)
\end{abstract}

Keywords: diabetes mellitus; nephrotic syndrome; primary immunodeficiency

Diabetes mellitus has long been considered a risk factor for recurrent infection; indeed it has been classified as a cause of secondary immunodeficiency by the World Health Organisation. ${ }^{12}$ We present a patient with a history of recurrent cutaneous and pulmonary infections, nephrotic syndrome, and an established diagnosis of type 1 diabetes who had unsuspected and unrecognised primary immunodeficiency.

\section{Case report}

A 31 year old male printer, in whom insulin dependent diabetes (IDDM) had been diagnosed in infancy, failed to improve on prophylactic antibiotics prescribed for recurrent skin and chest infections. There had been numerous admissions for incision and drainage of recurrent subcutaneous infections. In November 1997 he was admitted with dyspnoea and suspected chest infection not responding to oral antibiotic treatment. Peripheral oedema, glove and stocking sensory neuropathy, and bilateral proliferative retinopathy were noted on clinical examination. Urinalysis showed protein +++ in addition to glucose. Blood tests revealed a normochromic normocytic anaemia (haemoglobin $9.4 \mathrm{~g} / \mathrm{l}$ ), glucose $4.1 \mathrm{mmol} / 1$, $\mathrm{HbA} 1 \mathrm{c} 7.9 \%$, urea raised at $10.6 \mathrm{mmol} / 1$ (nor$\mathrm{mal}<7.0$ ), normal creatinine of $76 \mu \mathrm{mol} / \mathrm{l}$, and hypoalbuminaemia (albumin $31 \mathrm{~g} / 1$ ). Chest $x$ ray showed extensive right middle lobe consolidation, while thoracic computed tomography confirmed bronchiectasis and pulmonary oedema. On review of previous results from
1986, during an admission with pneumonia, a normal albumin and low total protein had been recorded but not investigated further.

Serum immunoglobulins were checked by chance by the admitting team and we were asked to advise when severe panhypogammaglobulinaemia was confirmed: IgA $<0.26 \mathrm{~g} / 1$ (normal 1-5), IgG $0.08 \mathrm{~g} / 1$ (normal 6.8-15.6), and $\operatorname{IgM} 0.17 \mathrm{~g} / 1$ (normal 0.5-2.8).

In conjunction with the 1986 results, a primary antibody deficiency was likely. Nephrotic syndrome was confirmed on a 24 hour urine collection: protein excretion $5.4 \mathrm{~g}, 4.3 \mathrm{~g}$ of this being albumin. No IgG was detected in this urine, there was no serum paraprotein, and lymphocyte immunophenotyping was normal. Overall, the immunological and radiological results were consistent with a diagnosis of common variable immune deficiency (CVID). The nephrotic state was most likely a result of diabetic nephropathy, especially with the other diabetic complications present (neuropathy and retinopathy). Creatinine clearance was normal at $102 \mathrm{ml} / \mathrm{min}$. Amyloidosis was considered in the context of recurrent infection; however, renal biopsy would not have altered management, so it was not performed. Intravenous immunoglobulin replacement therapy was deferred until we could be confident that infused IgG would not be wastefully lost in the urine.

Prophylactic antibiotics were prescribed, panretinal photocoagulation arranged, and an angiotensin converting enzyme inhibitor (ACEI) started. Repeat 24 hour urine collection after one month (January 1998) showed a dramatic response to ACEI, with protein excretion reduced to only $1 \mathrm{~g}$. A trial of intravenous immunoglobulin with serial measurement of serum and urine IgG was then performed (fig 1). At no time was IgG detected in the urine.

We established lifelong intravenous immunoglobulin treatment at three weekly intervals $(0.3 \mathrm{~g} / \mathrm{kg} / \mathrm{month})$. There have been no infective episodes in the subsequent 18 months, with trough IgG levels consistently $>7.0 \mathrm{~g} / 1$. Twenty four hour urine protein excretion remains suppressed and blood pressure well controlled. The major complication of intravenous immunoglobulin treatment has been deterioration in blood glucose control in the 24 hour period after each infusion, reflecting the fact that Sandoglobulin (Novartis) contains sucrose as a stabilising agent (10 g sucrose per $6 \mathrm{~g}$ Sandoglobulin). Our patient manages this by monitoring his blood glucose four times daily and increasing each Actrapid dose by $2-4$ units, leaving the nocturnal dose of Monotard 


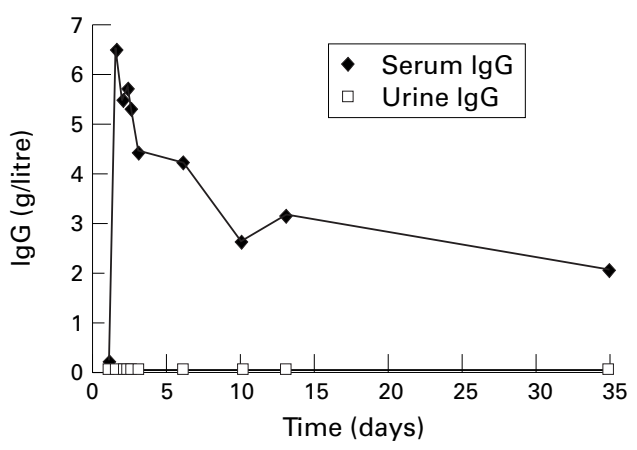

Figure 1 Serum and urine IgG responses (over 35 days of follow up) to a $24 \mathrm{~g}$ intravenous immunoglobulin infusion. An initial serum IgG bolus effect was followed by equilibration over two days, with subsequent decline consistent with the known half life of intravenous immunoglobulin. No IgG detected in the urine at any time.

unchanged. Using this regime his blood glucose (BM test) results remain below 12 $\mathrm{mmol} / \mathrm{l}$ following an infusion. Clinicians dealing with insulin dependent diabetic patients requiring intravenous immunoglobulin need to be aware of this effect on blood glucose control, and should encourage the use of blood glucose monitoring to guide increased insulin requirements over the subsequent 24 hours.

\section{Discussion}

The diagnosis of a primary immunodeficiency was considerably delayed in our patient, a common finding in CVID. ${ }^{3}$ IDDM was the major confounding factor in this case, with its well recognised but poorly understood association with recurrent infection. However, the nephrotic state with possible IgG leakage can also predispose to infection. This case is of interest because IDDM with nephrotic syndrome and CVID occurring together have not been reported before. A review of 291 patients with primary hypogammaglobulinaemia in the United Kingdom (240 with CVID, seven with thymoma, and 44 with Bruton's disease) in $1992^{4}$ found only two patients with maturity onset diabetes (both CVID), and none with type 1 diabetes.

Renal protein loss in diabetic nephropathy typically involves albumin, but can include larger molecules such as $\mathrm{IgG}^{5}$; however, the macromolecular forms of dimeric IgA and pentameric $\operatorname{IgM}$ are much less likely to be excreted. Our patient had panhypogammaglobulinaemia and despite heavy initial proteinuria at no time was immunoglobulin detected in the urine. This allowed us to provide adequate treatment for the antibody deficiency without leakage of infused IgG through the renal tract.

The low total protein result from 1986 suggested a problem at that stage, especially with the history of recurrent chest infections. This should have raised questions about what was missing from the serum. IgG levels would almost certainly have been low at that time. Unfortunately, bronchiectasis was present by the time the diagnosis was made, a condition potentially avoidable with earlier replacement immunoglobulin therapy.

This case highlights how easy it is to miss the diagnosis of hypogammaglobulinaemia despite the obvious infection history. All results need to be interpreted in the context of the case, so that important and treatable diagnoses are not delayed, with significant implications for the patient in the long term.

1 Pozzilli P, Leslie RDG. Infections and diabetes: mechanisms and prospects for prevention. Diabetic Med 1994;11:935and 41 .

2 World Health Organisation. Immunodeficiency: report of a scientific group. Technical Report Series 630. Geneva: WHO, 1978 .

3 Blore J, Haeney M. Primary antibody deficiency and diagnostic delay. BMF 1989;298:516-17.

4 Hermaszewski RA, Webster ADB. Primary hypogammaglobulinaemia: a survey of clinical manifestations and complications. $Q \mathcal{F}$ Med 1993;86:31-42.

5 Nakamura Y, Myers BD. Charge selectivity of proteinuria in diabetic glomerulopathy. Diabetes 1988;37:1202-11. 